\title{
Visión panorámica actual del Ingeniero de Planta en el proceso de fabricación del cemento
}

PERCY B. R. CASTILO NEYRA, Ingeniero AREQUIPA - PERU

\section{$R E S U M E N$}

La crisis energética y la subsecuente retracción de ventas en el mercado han demandado una mavor exigencia en el aspecto productivo del cemento, requiriéndose menores costos de producción y una mejor adecuación de la calidad del producto a las características de su empleo en obra.

En este articulo se enfoca las perspectivas que debe considerar el Ingeniero de Planta para el logro de estos objetivos. Se destaca la importancia y necesidad de la provección del proceso a las características del producto final, asi como la utilidad de un conocimiento adecuado de las materias primas.

Se mencionan otros factores que podrian considerarse igualmente como herramientas de trabajo para el Ingeniero de Planta, pero siempre bajo la consideración de que su mejor utilización dependerá fundamentalmente del criterio con que sean empleadas, por constituir cada fábrica un mundo diferente y particular.

\author{
$S U M M A R Y$
}

The energy crisis and subsequent retraction of sales on the market have demanded agreater exigency in the productive aspect of cement, requiring lower production costs and making the quality of the product more appropriate to the nature of its use in construction.

This article focuses on the perspectives which should be considered by the Plant Engineer in order to achieve these objetives. The importance and necessity of projecting the process to the characteristics of the end product are stressed, as well as the usefulness of an adequate knowledge of the raw materials.

Other factors which could also be considered as work tools of the Plant Engineer are mentioned, always taking into consideration, however, the fact that their best use will basically depend on the criterion with which they are put to use, since each factory constitutes a different world of its own.

\section{INTRODUCCION}

Debido fundamentalmente a la crisis energética, como causa primaria de motivación, a partir de la década de los 70 se han realizado notables e innumerables esfuerzos orientados a la disminución del consumo de energia, invertida en el proceso de fabricación, y la adecuación del producto a su empleo en la práctica en las condiciones técnicas y económicas más ventajosas.

Los logros en ambos aspectos son indiscutibles: E1 diseño de nuevos sistemas y equipos ha permitido disminuir al mínimo el consumo de energia en todas las etapas de fabricación, y la investigación de las caracteristicas intrinsecas del proceso han proporcionado un conocimiento amplio y profundo sobre los fenómenos físico-químicos involucrados, permitiendo un mayor aprovechamiento de las caracteristicas de las materias primas para producir a costos más bajos clinkeres o cementos de igual o mejor calidad.

Nuevos tipos de cementos han sido producidos, incluyendo adiciones de materiales con caracteristicas hidráulicas propias o combinadas, y se ha logrado una mejor adecuación de cada tipo de cemento a condiciones de emplco más convenientes y que ofrezcan mejores resultados. 
Sin embargo estos logros han sido alcanzados en términos generales, y se menciona referencias de consumos energéticos bajisimos en casos individuales. pero también son muchos los casos en los cuales no se obtienen los mismos o parecidos resultados, debido a una inadecuada aplicación de esta tecnología a casos particulares.

En este artículo se pretende dar una orientación de carácter general, en sentido teórico práctico, del enfoque que debe aplicar quien tiene a su cargo la dirección técnica de una planta, para el logro de mejores resultados individuales, tomando como base fundamental el hecho innegable de que cada fábrica de cemento representa, en sí, una realidad particular de características propias y especiales para cada caso. De tal forma, igual o mayor importancia que la calidad de las materias primas empleadas, los combustibles utilizados, y los sistemas y equipos de que se disponga, tendrán el criterio con el que estos elementos son combinados para el logro de los objetivos más ambiciosos, y que deben orientar el trabajo del Ingeniero de Planta.

Intencionalmente se ha procurado resistir a la tentación de profundizar en detalles técnicos. por no ser éste el objetivo del presente artículo.

\section{EL CRITERIO FUNDAMENTAL}

Las necesidades de optimización del proceso y la adecuación del producto a usos especificos han influido positivamente en el desarrollo de la Tecnologia del Cemento. y han motivado que se produzca un cambio en la mentalidad del Ingeniero de Planta, cuyos objetivos y actividades podrian ser enunciados en la siguiente forma: "La fabricación del cemento en planta debe ser orientada en función de las caracteristicas que se desean obtener en el producto final, a partir de materias primas de caracteristicas conocidas, y con el menor costo posible en el proceso de transformación".

Aparentemente, el tomar como factor prioritario la calidad del producto podria limitar algunas posibilidades de producción en condiciones óptimas de costos y rendimientos, pero sucede todo lo contrario. Tomando como punto de partida el conocimiento detallado de las materias primas que se emplean y los equipos para cada etapa del proceso de que se dispone. el cumplimiento del objetivo mencionado involucrará el aprovechamiento de todas las ventajas que resulten utilizables durante el proceso, y sólo existirá una línea óptima de producción bajo las condiciones dadas, con la cual se logren los mejores resultados, y a ella debe dirigirse el Ingeniero de Planta.

El mejoramiento de los parámetros de producción a partir de estas condiciones involucrará la implantación de sistemas o equipos que se determinen para cada caso y que resulten técnica y económicamente convenientes.

Estos conceptos pueden parecer obvios en teoria, pero para juzgar su actual objetividad en la práctica basta hacernos una simple pregunta: ¿Cuántos Ingenieros de Planta, especializados en el proceso de fabricación, conocen las caracteristicas del empleo del cemento en obra, la influencia de su composición fisico-química en su resistencia, estabilidad y durabilidad, su participación como importante constituyente del hormigón o sus posibilidades de aplicación en casos especiales? Seguramente no son muchos, y esto resulta negativo para los objetivos perseguidos, puesto que son frecuentes los casos en los cuales una modificación en la etapa final de fabricación o en la dosificación en obra, destinada a cubrir una deficiencia o mejorar una caracteristica del cemento, representa un costo negativo mucho mayor que quizás un importante ahorro o un brillante rendimiento alcanzado en alguna de las etapas de fabricación, pero realizado sin valorar la proyección necesaria y la integración en un objetivo final que debe caracterizar la marcha del proceso en planta. 


\section{EL PUNTO DE PARTIDA: MATERIAS PRIMAS}

La importancia de las materias primas es de carácter fundamental, dado que su disponibilidad y caracteristicas determinan desde la decisión de instalación. hasta el diseño de la planta en función de los procesos de transformación a los que será sometida y los cuales deberán ajustarse a las condiciones que resulten técnica y económicamente más rentables. El conocimiento por parte del Ingeniero de Planta de las caracteristicas de las materias primas, en forma detallada y apropiada, le brindará por lo general grandes posibilidades de introducir mejoras en el proceso, tomando en consideración que con el avance tecnológico y creación de nuevos sistemas, asi como la existencia de nuevas maquinarias y equipos. los criterios de decisión al construirse la planta, en el caso de fábricas en funcionamiento, tienen que haber variado, e incluso el conocimiento más complejo y completo de las materias primas que permiten los nuevos métodos y aparatos de análisis determinará nuevas perspectivas.

Tradicionalmente se juzga la calidad de las materias primas utilizadas en base a su composición quimica, características físicas tales como triturabilidad y molturabilidad, y contenido de elementos contaminantes considerados dañinos para el proceso o el producto final. Sin embargo, igual o mayor importancia para cada caso, se le debe adjudicar a su origen geológico, mineralogía y estado de cristalización, en forma individual y considerándolas como mezclas, bajo cuyas circunstancias tendrán un comportamiento en el cual la influencia mutua resultará determinante.

Cada especie mineralógica aporta de uno a varios óxidos al crudo, presenta una rigidez química del edificio reticular caracteristica y que representa un valor de la energia de activación que posee y dependiente de su origen y formación geológica a través del tiempo.

Imperfecciones en la red. estructuras defectuosas y desórdenes de cualquier otro tipo. aumentan la reactividad: igualmente los pscudosólidos y materiales mal cristalizados. o en estado de cristalización incipiente, tienen gran reactividad debido a la energia libre de sus estructuras.

También el estado de hidratación determinará que dentro del proceso térmico a que son sometidas, cuando el agua quimicamente combinada se evapore, deje espacios libres que debilitan la estructura y determinan mayor reactividad.

La aptitud a la cocción de los crudos producidos a partir de las materias primas dependerá en alto grado del estado de actividad de las especies presentes.

La influencia de los componentes minoritarios antiguamente considerados como impurezas, ha tomado nuevas e interesantes perspectivas desde el punto de vista del consumo de energia y su influencia en las caracteristicas del cemento: componentes considerados anteriormente como contaminantes indeseados, actualmente son convenientemente aprovechados para aspectos positivos.

Un aspecto de singular importancia se aprecia al analizar la gama de variedades en las cuales puede actuar el Ingeniero de Planta para lograr la optimización del proceso a su cargo, pudiendo optar en este sentido por adecuar las condiciones de operación a las caracteristicas que presente cl material en proceso. o a la inversa. efectuar modificaciones en las materias primas recurriendo incluso a aditivos o correctores adicionales, para adaptarlas a condiciones operativas que resulten más convenientes.

Es importante anotar, sin embargo, que las características consideradas tradicionalmente como las de mayor importancia son regulables durante las diferentes etapas del proceso, y con este objeto han sido diseñados los equipos y sistemas en la planta; en el caso de las características mineralógicas y estructurales de cada especie geológica, éstas no podrán ser modificadas para 
adecuarlas al proceso térmico de transformación, y se deberá tratar de aprovechar sus cualidades y evitar las que resulten perjudiciales, para lo cual es de fundamental interés e importancia que el Ingeniero de Planta conozca las materias primas y las posibilidades que se le presentan.

\section{EL CAMINO POR RECORRER: EL PROCESO}

Partiendo de materias primas de caracteristicas conocidas y habiendo determinado las requeridas en el cemento como producto final. la transformación que se realiza durante el proceso involucra gran cantidad y variedad de operaciones. que se realizarán de acuerdo a una planificación desarrollada en el diseño de la planta.

La exigencia de efectuar esta transformación con una inversión minima de costos. pero cumpliendo con las condiciones pre-establecidas referentes a calidad y cantidad de producción. y las exigencias referentes a contaminación ambiental, seguridad ambiental, ambiente laboral, etc. ha deteminado que el proceso se torne cada vez más complejo.

Dada la incidencia de todos estos factores en el aspecto económico se ha prestado especial interés en la creación y modificación de equipos. y la modernización de métodos y sistemas. proyectando el avance de la ciencia y la técnica a este campo: los resultados han sido admirables, y durante los últimos años el desarrollo de la Tecnología de Fabricación del Cemento ha tenido un gran impulso.

Si bien estas circunstancias han provocado que el trabajo del Ingeniero de Planta se torne más complejo, a la vez le han proporcionado valiosas herramientas para el cumplimiento de su labor. exigiendo asimismo de su parte un dinamismo y una necesidad y exigencia de continua actualización de conocimientos profesionalmente muy saludable.

Cada planta de cemento constituye un mundo diferente y complejo de caracteristicas y personalidad propia, y cada etapa del proceso, considerada bajo la misma óptica, un sub-mundo que debe ser enfocado en forma individual pero también como parte integrante de un engranaje de funcionamiento con un objetivo final común. Son muchas las comparaciones que podrian utilizarse para representar el trabajo de la planta, por ejemplo: Un motor eléctrico, en cuyo funcionamiento cada una de las piezas tendrá una función e importancia individual, pero que para su adecuada marcha y producción, en condiciones económicas óptimas. requiere el cumplimiento integral de la labor de cada una de las piezas para la cual han sido incluidas. Siendo tan amplia y compleja la marcha de la planta, resulta necesario simplificar su control y dirección, y para ello un método que resulta muy funcional es el de fijación de objetivos en forma individual para cada etapa del proceso, manteniendo la correlación necesaria entre ellas, para asegurar el objetivo final.

La delimitación de etapas que han de considerarse estará determinada por las operaciones que se realizan en planta y la planificación que se ajuste a cada realidad particular, permitiendo facilitar un permanente y efectivo control de funcionamiento.

Cada etapa presentará caracteristicas propias de acuerdo a las operaciones que se realicen y los sistemas y equipos que se empleen, pero que en lo referente a los objetivos perseguidos presentarán un denominador común: la adecuación del producto a la siguiente etapa del proceso, $\mathrm{y}$ su proyección a las características del producto final.

Habrá etapas que involucren un espectro más amplio y que por su incidencia más significativa en los costos de producción revistan mayor importancia y requieran mayores medios y atención; pero para efectos de carácter general, será de fundamental importancia que se parta del principio de que todos los objetivos individuales deben cumplirse, pues en el proceso todas las fases están inter-relacionadas y las variaciones de las condiciones fijadas para una etapa considerada de 
menor importancia dificultará y podría incluso impedir el cumplimiento de los objetivos de las etapas subsecuentes y, por ende, el objetivo final.

La fijación de objetivos deberá ser realizada con mucho criterio y ajustado a la realidad, pues influirá radicalmente en las posibilidades económicas de producción del producto deseado. Objetivos demasiado cómodos ocasionarán no solo el desaprovechamiento de recursos valiosos, con el consiguiente perjuicio económico, sino además dificultará y entorpecerá el desarrollo del proceso mismo, pues es incontrastable que la única forma adecuada de hacer efectivo un proceso, y obtener los resultados esperados, es realizarlo en forma integralmente correcta. En otro aspecto, propicia un ambiente laboral muy negativo y limitado en cuanto a aporte de iniciativas y sugerencias del personal, cuya experiencia y contanto directo con los equipos hacen estas actitudes altamente deseables y provechosas.

En contraposición, objetivos demasiado ambiciosos también ocasionarán efectos contraproducentes: alteración de las condiciones idóneas del proceso, que exige mayor estabilidad de condiciones en el tiempo antes que rendimientos elevados pero que no puedan ser mantenidos y que provoquen alteraciones perjudiciales, así como un ambiente laboral demasiado exigente y con demasiada presión sobre el personal con resultados indeseados.

La planificación de objetivos deberá considerar absolutamente todos los factores que puedan influenciar su cumplimiento, y por tal razón involucra una gran variedad de aspectos cuya responsabilidad y control no se encuentra normalmente a cargo del Ingeniero de Planta; por este motivo debe ser realizada con participación de todos los sectores que se encuentren comprometidos en el área Técnica y en coordinación con el Sector Administrativo.

La complejidad de su planificación y el control permanente que es necesario mantener posteriormente sobre todos los aspectos considerados, determinan que este área se preste especialmente a la implantación de un sistema de ordenador, lo cual permitirá un importante aporte en su control de funcionamiento y caracterización, y consecuentemente, mejores resultados técnicos y económicos.

En el proceso se presentan una multitud de factores que influyen en su realización, rendimiento y productividad, pero el de mayor importancia en cuanto a resultados se refiere, será el criterio y la decisión del Ingeniero de Planta encargado de su conducción.

\section{EL OBJETIVO FINAL: CEMENTO}

En una determinada zona de influencia, el tipo de cemento requerido y las características especiales que éste deba satisfacer, estarán en función de las condiciones particulares que se presenten para su empleo en obra. La fábrica que haya sido proyectada para cubrir las demandas del mercado en esta zona, orientará su producción hacía un cemento que cumpla con estos requerimientos, en la medida que lo permitan la calidad de las materias primas y su disponibilidad en la zona, la caracterización de sus instalaciones, y los sistemas y métodos empleados en el proceso de fabricación.

La retracción de mercado a niveles locales e internacional, causada por la crisis energética, ha cambiado la mentalidad del fabricante acostumbrado por lo general a mercados con mayor demanda que oferta, y se ha visto sometido a una exigencia interna y externa. La primera consistente en la disminución de costos de producción y mantenimiento de condiciones de calidad que le permitan competir técnica y económicamente en el mercado, y la segunda por la necesidad de proyectarse a la ampliación del campo de aplicación del cemento. ya sea produciendo nuevos tipos del mismo. o impulsando su empleo en usos no tradicionales. 
Las características que deben cumplir, en lo referente a la calidad de los cementos producidos, pueden referirse en términos generales a su resistencia mecánica, durabilidad y estabilidad de volumen. El cumplimiento de las condiciones mínimas requeridas en estos aspectos demandará la adecuación de las caracteristicas de producción a las exigencias a que estará sometido el cemento en la práctica, y por esta razón resulta de imperiosa necesidad que el Ingeniero de Planta se proyecte al campo del uso del cemento, e incluso a la Tecnologia del Hormigón, lo cual le permitirá un criterio más amplio no sólo para cumplir exigencias, sino para proyectar y efectuar cambios que permitan una mejor adecuación del proceso productivo y minimizar los costos de producción.

En la práctica, en el empleo del Hormigón, existe la tendencia del usuario de achacarle todos los problemas que se presentan al cemento y su calidad, lo cual sólo representa uno de los numerosos factores que influyen en la calidad del Hormigón y quizás el único sometido a un exigente control de calidad; sin embargo cuando no se tiene el conocimiento de causa que deseche esta posibilidad, se pueden cometer serios errores al tratar de compensar en la planta defectos inexistentes y que sólo provocarán un costo mayor de producción y quizás verdaderos problemas de diferente origen y repercusión.

De todas maneras, el criterio del Ingeniero de Planta debe dirigirse a asegurar y mantener una calidad del cemento bajo parámetros establecidos, anteponiendo este objetivo a los volúmenes de producción y rendimientos energéticos, los cuales serán más fáciles de superar una vez alcanzado el primero, con mejores resultados globales y mayores niveles técnicos.

Un campo que requiere la mayor atención por su gran significado económico y técnico es el de Cemento con Adiciones, llámense éstas: puzolanas, cenizas, escorias, o fillers calizos. La adición de éstos materiales puede ser considerada desde ambos aspectos: técnicamente, por constituir estas adiciones elementos que le confiere al cemento características especiales que permiten su aplicación en obras, en las cuales el uso de cemento portland puro, debido a sus conocidas deficiencias en algunos aspectos se presenta problemática; económicamente, al reemplazar en el cemento un porcentaje de clínker, con el consiguiente ahorro energético y económico.

Las condiciones fabricación y empleo de estos cementos ofrecerán ciertas variantes respecto a las del cemento portland puro. El aprovechamiento de las condiciones favorables que permitan en la molienda la presencia de adiciones, la producción de cementos con características que permitan su máximo aprovechamiento en la práctica, la compensación de las deficiencias que pudiesen presentar, la determinación de las mejores características que se deban procurar en el clínker para este tipo de cementos, etc., son algunos de los temas que deben ser motivo del mayor interés del Ingeniero de Planta para lograr el máximo aprovechamiento en este campo.

\section{LA INVESTIGACION APLICADA}

La necesidad de producir cementos de mejor calidad y con menor consumo de energia por las razones conocidas derivadas de la crisis energética, y cl adelanto tecnológico actual, ha favorecido la realización y publicación de innumerables trabajos de investigación ubicados dentro del campo de la fabricación y usos del cemento.

Esta realidad nos permite disponer de una considerable bibliografia de consulta para los problemas que se presenten en cada planta, o implantar mejoras en el proceso de fabricación, lo cual resulta de invalorable utilidad, siempre que sea utilizado con el criterio y adecuación necesaria para cada realidad particular, pudiendo en caso contrario producir efectos absolutamente opuestos a los deseados.

Cada fábrica constituye una realidad diferente, y lo que en un caso puede resultar altamente 
beneficioso, aplicado en otras circunstancias, bajo los mismos principios podria significar todo lo contrario.

Son muchos los casos en los cuales algún fabricante ha expresado sus dudas acerca de los resultados experimentados en otras plantas con éxito, por no haber obtenido resultados similares en la propia, pero esto solo significa que este sistema es inaplicable para la suya, o quizás podria resultar aún más beneficioso que en la misma planta referida, pero aplicado bajo criterio propio y adecuado a las caracteristicas de su realidad particular.

Otro aspecto, igualmente de gran importancia, es la diferencia que existe en casi la generalidad de los casos, entre las condiciones de laboratorio e incluso en plantas piloto, y las que se dan a nivel industrial en la planta de fabricación.

Esto provoca que muchas de las experiencias realizadas a nivel industrial basadas en conclusiones de trabajos de investigación hehcos en laboratorio no produzcan los resultados esperados, lo cual no significa una incompatibilidad entre ambos aspectos, sino simplemente que no se ha efectuado en la forma adecuada la interpretación de las conclusiones referidas, y su aplicación al campo práctico se ha hecho sin el criterio de objetividad necesario. Por estas razones y por la razón de ser de la Ingeniería misma, se impone y justifica que en todas las fábricas de cemento se realice Investigación.

Dependiendo de las facilidades y disponibilidad de equipos en planta y laboratorio se deberá necesariamente efectuar una planificación de actividades y pruebas a nivel de laboratorio y planta, cuyas conclusiones permitirán en forma inmediata el conocimiento de aspectos de carácter interno, y ofrecerán siempre nuevas perspectivas para el mejoramiento de los parámetros de producción. Esta será además la mejor manera de acceder y familiarizarse con las actividades que se realizan en el campo de la Investigación de alto nivel cientifico en temas relacionados con la Tecnología del cemento, y que servirá para mejor interpretación de sus resultados, así como mejor nivel de conocimientos del proceso.

Puede existir escasa disponibilidad de equipos que aparentemente impidan o limiten la capacidad de investigación, pero esto no es causa justificatoria para no realizarla, pues se puede efectuar Investigación a todos los niveles, siendo quizás de mayor importancia los equipos de que se disponga, el interés que se invierta en realizarla, además de una adecuada Planificación y un análisis realista de los resultados obtenidos.

Finalmente el mejor laboratorio para las condiciones de una fábrica lo constituye la planta misma, siempre que se actúe con la sensatez y planificación necesaria para evitar resultados que pudiesen dañar los equipos o alterar los objetivos de producción. 